\title{
Refractory Multi-inflammatory Syndrome in a two weeks old neonate with COVID-19 Treated Successfully with Intravenous Immunoglobulin, Steroids and Anakinra
}

\section{Samar Magboul ( $\sim$ smagboul1@hamad.qa )}

Hamad Medical Corporation https://orcid.org/0000-0002-7263-565X

Ahmed Khalil

Hamad Medical Corporation

Ahmad Alshami

Hamad Medical Corporation

Mohamed Alaido

Hamad Medical Corporation

Abdulla Alhothi

Hamad Medical Corporation

khaled Ellithy

Hamad Medical Corporation

Mohamed Al Amri

Hamad Medical Corporation

Manasik Hassan

Hamad Medical Corporation

\section{Case Report}

Keywords: Neonatal COVID-19, SARS-CoV-2, multisystem inflammatory syndrome of children MIS-C, pediatric multi-system inflammatory syndrome PIMS.

Posted Date: August 24th, 2020

DOl: https://doi.org/10.21203/rs.3.rs-60510/v1

License: (1) This work is licensed under a Creative Commons Attribution 4.0 International License. Read Full License 


\section{Abstract}

Background: World Health Organization (WHO) and other Health officials alert clinicians about a rare but severe inflammatory condition seen in children and linked to Corona Virus Disease 2019(COVID-19). The WHO is describing the condition as a multisystem inflammatory syndrome in children (MIS-C) and is recommending clinicians to report those cases to get a better understanding of the disease and clinicians can learn more.

Case presentation: We are reporting the clinical course of the youngest case of COVID-19 related MIS-C; a two-week-old term neonate with COVID-19 infection and features suggestive of MIS-C , managed with intravenous immunoglobulin (IVIG), pulse steroid, and interleukin-1 inhibitor (Anakinra).By reviewing the literature, our baby is the first neonatal case who has been diagnosed with MIS-C.

Conclusion: COVID-19 infection in pediatrics are likely to present with a mild course; however, some may develop a hyper-inflammatory syndrome. Pediatricians should be aware of such presentation, the clinical course, the management modalities, and inform parents and caregivers about common signs and symptoms. Anakinra may consider as effective second agent in (IVIG and steroid-refractory pediatric cases).

\section{Background}

The clinical course, progression, and outcome of COVID-19 disease in pediatrics are milder and less sever than adult. They have lower rates of hospitalization and death as well ${ }^{1-5}$. Multi-system inflammatory syndrome in children (MIS-C), which is called pediatric multi-system inflammatory syndrome (PMIS) as well, is a newly recognized, possibly serious illness in children that is recently connected to COVID-19 infection. It appears to be a late complication of COVID-19 infection, even though some patients were labeled with this complication without testing positive for sever acute respiratory syndrome corona virus -2(SARS-CoV-2). Since March 2020, many of the UK and USA pediatricians and in many other countries in Europe during the coronavirus disease 2019 (COVID-19) pandemic, started to notice and report children presenting with fever and multi-system inflammation. The presentations were variable and included children who had features similar to those of Kawasaki disease, streptococcal / Staphylococcal toxic shock syndrome, Sepsis, probably in relation to SARS-CoV-2 infection ${ }^{6-9}$. Children who were critically ill needed intensive care unit admission ${ }^{10}$.

To date, there are suggestions for diagnosing MIS-C based on case definition. World Health Organization(WHO) , Centers for disease control and prevention of United States (CDC-USA) and Royal college of pediatrics and child health of United Kingdom(RCPCH-UK) proposed the symptoms of (persistent fever, Kawasaki like features and dysfunction of one or more organs), laboratory investigations showing signs of inflammation, as the main criteria to diagnose MIS-c7, 11.

\section{Case Presentation}


A two weeks old female baby, born spontaneously at full term with a birth weight of $3.2 \mathrm{~kg}$ in the maternity unit at the hospital. She presented with neonatal fever and admitted to pediatrics inpatient in May 2020. Initial laboratory workup was done to rule out neonatal sepsis, and she had full sepsis workup done for her, included Lumbar Puncture, at the third day of admission she was checked for COVID-19 polymerase chain reaction (PCR) and found to be positive, though she did not have any contact with COVID-19 patient, both parents were screened with a nasal swab after her diagnosis and were negative. After that, she was shifted to pediatrics COVID-19 inpatient area in the main and only pediatrics COVID-19 institution and stayed there for nine days, where her clinical and laboratory conditions were strictly observed.

The baby was continuously spiking high-grade fever throughout her stay with baseline tachycardia; although she clinically and hemodynamically continued to be stable, her inflammatory markers were increasing (table 2), declaring a state of acute inflammation. She was conscious alert and active and did not have any respiratory symptoms and was having normal oxygen saturation on room air despite her chest $x$-ray showing bilateral lungs infiltrates (pneumonitis), when consulted pulmonologist advised for starting Antibiotics. However, her clinical examination only revealed mild hepatosplenomegaly with no skin rash, lymph nodes enlargement, or Kawasaki disease signs. She had multiple laboratory investigations aiming to discover the source of infection and the degree of inflammation.

Complete blood counts (CBCs) were showing lymphocytic leukocytosis with (total WBC $31600 / \mu \mathrm{L}$ ), she had normocytic normochromic anemia with hemoglobin level dropping up to (8.7 g/dL), With mild thrombocytopenia of $\left(124 \times 10^{\wedge} 3 / \mu \mathrm{L}\right)$. Her C-reactive protein (CRP) was steadily rising from (6 to $84 \mathrm{mg} / \mathrm{dl})$, hypoalbuminemia $(23 \mathrm{~g} / \mathrm{l})$, hyponatremia $(127 \mathrm{mEq} / \mathrm{L})$, and her lactic acid reached up to $(6.4 \mathrm{mmol} / \mathrm{l})$ with normal $\mathrm{PH}$ and Kidney function. Liver functions showed a mild increment in liver transaminases, aspartate aminotransferase (AST) and alanine aminotransferase (ALT), were doubled with normal coagulation(table 2). All her cultures came back negative with CSF cell count showing increased RBC counts -deemed traumatic, and we decided to do an ultrasound head, which came to be normal.

The baby was screened again after first positive COVID-19 PCR on two consecutive days, eighth \& ninth, and the results were negative COVID-19 PCR and other respiratory virus's nasal swabs(table 2). When she continued to be febrile and tachycardic with increasing inflammatory markers and a drop in her hemoglobin, Baby was transferred to the main hospital in the pediatrics inpatient unit in an isolation room to complete the investigation as multisystem inflammatory syndrome of children (MIS-C) was suspected. Her ferritin level markedly raised (1773 ug/l), Troponin, and her Natriuretic peptide test (proBNP) were both increased (table 2),cardiac wise she had an Echocardiogram which was done twice, and it was showing normal coronary arteries and contractility. With her persistent tachycardia and low hemoglobin, the primary physician and upon pediatrics hematology consult both agreed to transfuse her packed red blood cells. On the same day, pediatrics rheumatologist reviewed the baby and advised to start (IVIG two grams per kilogram per dose)(table1),along with Aspirin high dose of $75 \mathrm{mg}$ per dose every 6 hours per day, Same day she was given one dose of Dexamethasone(table 1)then commenced on 
Methylprednisolone as pulse steroid $(30 \mathrm{mg} / \mathrm{kg})$ once daily for three days with tapering dose over the next days(table 1). After IVIG infusion, the baby continued to have high inflammatory markers; then, it was decided by a multidisciplinary team to admit her to the pediatric intensive care (PICU) unit to give the second line therapy, immunomodulatory therapy (Anakinra) as a refractory case.

Dose of Anakinra was (2mg/kg) loading dose(table1), followed by a continuous infusion of $(0.02 \mathrm{ml} / \mathrm{kg} / \mathrm{hour})^{12}$. Efficacy and safety-wise were the reasons to give continuous infusion, not subcutaneous (SC) injection, as there is no recommended dose for this age group as SC injection. She received Anakinra on day 17 of illness and completed 9 days then was stopped. She was kept on antibiotics (Piperacillin/Tazobactam)(Table 1) for presumptive pneumonitis for 10 days and as pediatrics infectious diseases services were involved early, Baby was not started on any other COVID-19 medications, and especially she did not have any respiratory symptoms.

Baby was then re-spiked low grade fever on day 34 of admission and after around 24 hours from discontinued Anakinra, her primary team decided to give her a second dose of Intravenous immunoglobulins IVIG, unfortunately no laboratory investigations were taken before starting it, then was observed for three more days with inflammatory markers went back to normal . She was discharged home after total of five weeks of hospitalization in good general condition, she was then followed up by pediatric rheumatology and pediatric cardiology team along with general pediatrics . Echocardiogram was also repeated and continued to be normal.

\section{Discussion}

Our case report involving hospitalized neonatal case, two weeks old with MIS-C, which is the youngest case in State of Qatar and according to our knowledge through all the literature as well. Our case fulfils the criteria of Pediatric multisystem inflammatory syndrome associated with COVID-19 and the case definition that has been recommended by different sources such as New York State Department of Health (NYSDOH) and European Centre for Disease Prevention and Control (ECDC) ${ }^{7,11}$. Only one suspected neonatal case was reported in a research study group, involved with the age Group ( 0 to 5 years of age) in New York. The baby presented at 14 and 28 days of age with fever and left breast cellulitis. Echocardiogram Showed good ventricular function and unremarkable coronary arteries. Two molecular tests for SARS-CoV-2 were negative. The discharge diagnoses were cellulitis, and shock ${ }^{12}$.

As in different studies, our MIS-C case followed the peak of infection by almost two weeks, which support that this syndrome is probably a post infectious inflammatory process related to Covid-19 ${ }^{12,13}$. A profound inflammatory response resulting in Acute Respiratory Distress Syndrome (ARDS) and multiorgan failure seems to be an essential component of the critical illness associated with COVID-19. Some critically ill cases will manifest shock and cardiac dysfunction, probably due to cytokine storm (CS) resulting from the host response to viral infection ${ }^{14}$. Interleukin -6 (IL-6) \& Interleukin 1 beta (IL-1 $\beta$ ) levels are elevated in patients with severe COVID-19; however, fewer data have been reported to date in COVID19 patients regarding IL-1 $\beta^{15}$. High inflammatory markers like C-reactive protein(CRP)level and other 
abnormal parameters that mentioned in the presentation of our case explain that this inflammatory syndrome is probable mediated by IL-6. Different immunomodulatory therapies have been discussed and tried to manage the inflammatory response such as Tocilizumab and Anakinra ${ }^{9,16}$.

Management remains difficult; most cases have been treated as atypical Kawasaki Disease with additional supportive care as needed. Considering the rarity and complexity of the syndrome, it would be essential to establish and coordinate to guide diagnosis, treatment, and follow-up. Our case received IVIG, Pulse steroid, then Anakinra. The decision regarding giving Anakinra not Tocilizumab for our baby was initiated, Since Anakinra is used to treat patients with neonatal-onset multisystem inflammatory disease (NOMID) ${ }^{17}$; it is known to be safe for use in neonates, infants as well as children and adults. Moreover there was no specific dose and safety profile regarding Tocilizumab use in this age group. Till more data are available, the routine use of Tocilizumab and Anakinra in patients with severe or life- threatening COVID-19 is not recommended. Nevertheless, the use of these medications may be considered, in consultation with pediatric Infectious Diseases, Immunology, and pediatric Rheumatology in conjunction with the patient's primary team in patients with severe disease and clinical deterioration. There are ongoing Clinical Trials, for example, NCT02735707, NCT04339712, NCT04330638, NCT04324021. A multicenter clinical trial in the United States is being designed to test the use of Anakinra in adults with COVID-19.

\section{Conclusion}

SARS-CoV2 infections in pediatrics are likely to present with a mild course; however, some may develop a hyper-inflammatory syndrome. Pediatricians should be aware of such presentation, the clinical course, the management modalities, and inform parents and caregivers about common signs and symptoms. Anakinra may consider as effective second agent in (IVIG and steroid-refractory pediatric cases).

\section{List Of Abbreviations}

HMC: Hamad Medical Corporation

HGH : Hamad General hospital

COVID-19 : Corona Virus Disease 2019

MIS-C: multisystem inflammatory syndrome of children

SARS-CoV-2: sever acute respiratory syndrome corona virus -2

WHO: World Health Organization

CDC: Centers of diseases prevention and control -United States

RCPCH: Royal college of pediatrics and child health-United Kingdom 
PCR: Polymerase chain reaction

CBC: complete blood count

WBC: white cell count

PCT: procalcitonin

CRP: C-reactive protein

ESR: erythrocyte sedimentation rate

LFT: liver function test

NT- pro BNP: Natriuretic peptide test

IL-6: Interleukin -6 test

IL-1 $\beta$ : Interleukin 1 beta

IVIG: intravenous immunoglobulin

Mg: milligrams

Kg: Kilograms

ARDS: Acute Respiratory Distress Syndrome

NYSDOH: New York State Department of Health

ECDC: European Centre for Disease Prevention and Control

\section{Declarations}

\section{Ethical Approval:}

This case report received Ethical approval from the medical research committee at Hamad Medical Corporation (MRC-04-20-601).

\section{Consent for publication}

Waiver of signed informed Consent for this publication was obtained.

\section{Availability of Data and materials:}

The data sets during and/or analyzed during the current study available from the corresponding author on reasonable request. 


\section{Declaration Competing interests:}

Authors declare that they have no competing interests and no potential conflicts of interests with respect to the research, authorship, and /or publication of this article.

\section{Funding:}

This study did not receive any specific funding.

\section{Authors' contributions:}

SA, AK and MH has conceptualized and designed the ,analyzed and interpreted patient's data, drafted the initial manuscript, Reviewed and revised the manuscript.

AAS, MA and MAA contributed in writing manuscript and analyzing patient's data

AA and KE have monitored the case course analyzed, interpreted the patient's data and reviewed the manuscript.

All authors read and approved the final manuscript as submitted and agree to be accountable for all Aspects of the work.

\section{Acknowledgment:}

The Authors are sincerely thankful to Dr. Fatima Mehsin and Dr. Buthaina Aladba for their valuable contribution in this case.

\section{References}

1. Rosenberg ES, Dufort EM, Blog DS, et al. COVID-19 Testing, Epidemic Features, Hospital Outcomes, and Household Prevalence, New York State-March 2020 [published online ahead of print, 2020 May 8]. Clin Infect Dis. 2020;ciaa549. doi:10.1093/cid/ciaa549

2. Dong, Y., Mo, X., Hu, Y., Qi, X., Jiang, F., Jiang, Z., \& Tong, S. (2020). Epidemiology of COVID-19 among children in China. Pediatrics, 145(6).

3. Zhou, F., Yu, T., Du, R., Fan, G., Liu, Y., Liu, Z., \& Guan, L. (2020). Clinical course and risk factors for mortality of adult inpatients with COVID-19 in Wuhan, China: a retrospective cohort study. The lancet.

4. Kim L, Whitaker M, O'Halloran A, et al. Hospitalization Rates and Characteristics of Children Aged $<18$ Years Hospitalized with Laboratory-Confirmed COVID-19 - COVID-NET, 14 States, March 1-July 25, 2020. MMWR Morb Mortal Wkly Rep 2020;69:1081-1088. DOI: http://dx.doi.org/10.15585/mmwr.mm6932e3external icon.

5. Coronavirus Disease 2019 in Children - United States, February 12-April 2, 2020. MMWR Morb Mortal Wkly Rep 2020;69:422-426. DOI: http://dx.doi.org/10.15585/mmwr.mm6914e4external icon. 
6. Jones, V. G., Mills, M., Suarez, D., Hogan, C. A., Yeh, D., Segal, J. B., \& Mathew, R. (2020). COVID-19 and Kawasaki disease: novel virus and novel case. Hospital Pediatrics, 10(6), 537-540.

7. European Centre for Disease Prevention and Control. Paediatric inflammatory multisystem syndrome and SARS-CoV-2 infection in children - 15 May 2020. ECDC: Stockholm; 2020.

8. Riphagen, S., Gomez, X., Gonzalez-Martinez, C., Wilkinson, N., \& Theohari's, P. (2020). Hyper inflammatory shock in children during COVID-19 pandemic. The Lancet, 395(10237), 1607-1608.

9. Royal College of Pediatrics and Child Health. Guidance: pediatric multisystem inflammatory syndrome temporally associated with COVID-19. 2020.

https://www.rcpch.ac.uk/resources/guidance-paediatric-multisystem-inflammatory-syndrometemporally-associated-covid-19-pims ,accessed 13 July 2020

10. Whittaker E, Bamford A, Kenny J, et al. Clinical Characteristics of 58 Children With a Pediatric Inflammatory Multisystem Syndrome Temporally Associated With SARS-CoV-

2. JAMA.2020;324(3):259-269. doi:10.1001/jama.2020.10369

11. Centers for Disease Control and Prevention (CDC) https://emergency.cdc.gov/han/2020/han00432.asp accessed 13 July 2020.

12. Dufort, E. M., Koumans, E. H., Chow, E. J., Rosenthal, E. M., Muse, A., Rowlands, J., ... \& Udo, T. (2020). Multisystem inflammatory syndrome in children in New York State. New England Journal of Medicine, 383(4), 347-358.

13. Verdoni, L., Mazza, A., Gervasoni, A., Martelli, L., Ruggeri, M., Ciuffreda, M., \& D'Antiga, L. (2020). An outbreak of severe Kawasaki-like disease at the Italian epicentre of the SARS-CoV-2 epidemic: an observational cohort study. The Lancet.

14. Ye, Q., Wang, B., \& Mao, J. (2020). The pathogenesis and treatment of theCytokine Storm'in COVID19. Journal of infection, 80(6), 607-613.

15. Huang, C., Wang, Y., Li, X., Ren, L., Zhao, J., Hu, Y., \& Cheng, Z. (2020). Clinical features of patients infected with 2019 novel coronavirus in Wuhan, China. The lancet, 395(10223), 497-506.

16. Zhang, W., Zhao, Y., Zhang, F., Wang, Q., Li, T., Liu, Z., \& Zeng, X. (2020). The use of anti-inflammatory drugs in the treatment of people with severe coronavirus disease 2019 (COVID-19): The experience of clinical immunologists from China. Clinical Immunology, 108393.

17. Science direct https://www.sciencedirect.com/topics/pharmacology-toxicology-and-pharmaceuticalscience/anakinra accessed 13 July 2020

\section{Tables}

Due to technical limitations the Tables are available as a download in the Supplementary Files.

\section{Supplementary Files}

This is a list of supplementary files associated with this preprint. Click to download. 
- Tables.pdf

Page 9/9 